\title{
Unexpected community-acquired co-infections during an ongoing pandemic: (one) too many diagnoses at once
}

\author{
David Manuel Cordas dos Santos ${ }^{1,2,3} \cdot$ Laura Fischer $^{1} \cdot$ Michael von Bergwelt-Baildon $^{1,3} \cdot$ Elham Khatamzas $^{1,4}$
}

Received: 6 May 2021 / Accepted: 20 June 2021 / Published online: 3 July 2021

(c) The Author(s) 2021

In November 2020, a 44-year-old man presented to our hospital with a two-week history of flu-like symptoms, night sweats and swollen lymph nodes. The patient stayed in an emergency shelter, reported significant regular alcohol intake (min. 20 units/day) and nicotine abuse (35 pack years). He was febrile, tachypnoeic with no neurological signs. Physical examination was remarkable for generalized peripheral lymphadenopathy and skin lesions (Fig. 1A). Blood film demonstrated leucocytosis with $63 \%$ blasts with absolute neutropenia. Histopathological examination of the skin lesions revealed chloroma as an extramedullary manifestation (Fig. 1B/C). Bone marrow examination confirmed the diagnosis of acute monoblastic leucaemia (Fig. 1D/E). Cytoreductive regimen with hydroxyurea was commenced.

Admission blood cultures were positive for Listeria monocytogenes and Streptococcus mitis. Viral screening for SARS-CoV-2, HIV and Hepatitis B/C was negative. Antimicrobial regimen with piperacillin-tazobactam and caspofungin was commenced with reduction in inflammatory markers (CRP 38 to $29 \mathrm{mg} / \mathrm{dl}$, procalcitonin 42 to $7 \mathrm{ng} / \mathrm{ml}$, interleukin-6 2625 to $63 \mathrm{pg} / \mathrm{ml}$ ). On day 4, the patient deteriorated rapidly progressing to septic shock and acute respiratory distress syndrome requiring multiorgan support. CT chest images showed dramatic progression of pulmonary changes (Fig. 2). In repeat microbiological investigations, surprisingly Legionella pneumophila was detected in urine and endotracheal secretions by antigen and PCR, respectively, suggestive of previously undiagnosed community-acquired Legionellosis. Due to absence of pulmonary symptoms, respiratory specimens had not been collected on admission. Legionella testing of water systems within our unit was negative. Local health protection agency was notified but investigations could not identify sources for either of two pathogens. Despite maximal treatment including escalation of antimicrobial regimen to meropenem and moxifloxacin on day 5 , the patient's condition deteriorated further $[1,2]$. Life-extending measures were terminated on day 9 .

Polymicrobial life-threatening infections are a known feared complication of hematological malignancies particularly during active immunosuppressive treatment [3-5]. However, the unique combination of community-acquired infections in this case, highlights the importance of environmental, behavioral and socio-economic risk factors as well as continued re-evaluation of differential diagnosis and therapy in managing these complex cases.

Elham Khatamzas

Elham.khatamzas@med.uni-heidelberg.de

1 Department of Medicine III, LMU University Hospital, Marchioninistraße 15, Munich 81377, Germany

2 Cancer- and Immunometabolism Research Group, LMU Gene Center, Munich, Germany

3 German Cancer Consortium (DKTK), partner site Munich, and German Cancer Research Center (DKFZ), Heidelberg, Germany

4 COVID-19 Registry of the LMU Munich (CORKUM), LMU University Hospital, Munich, Germany 

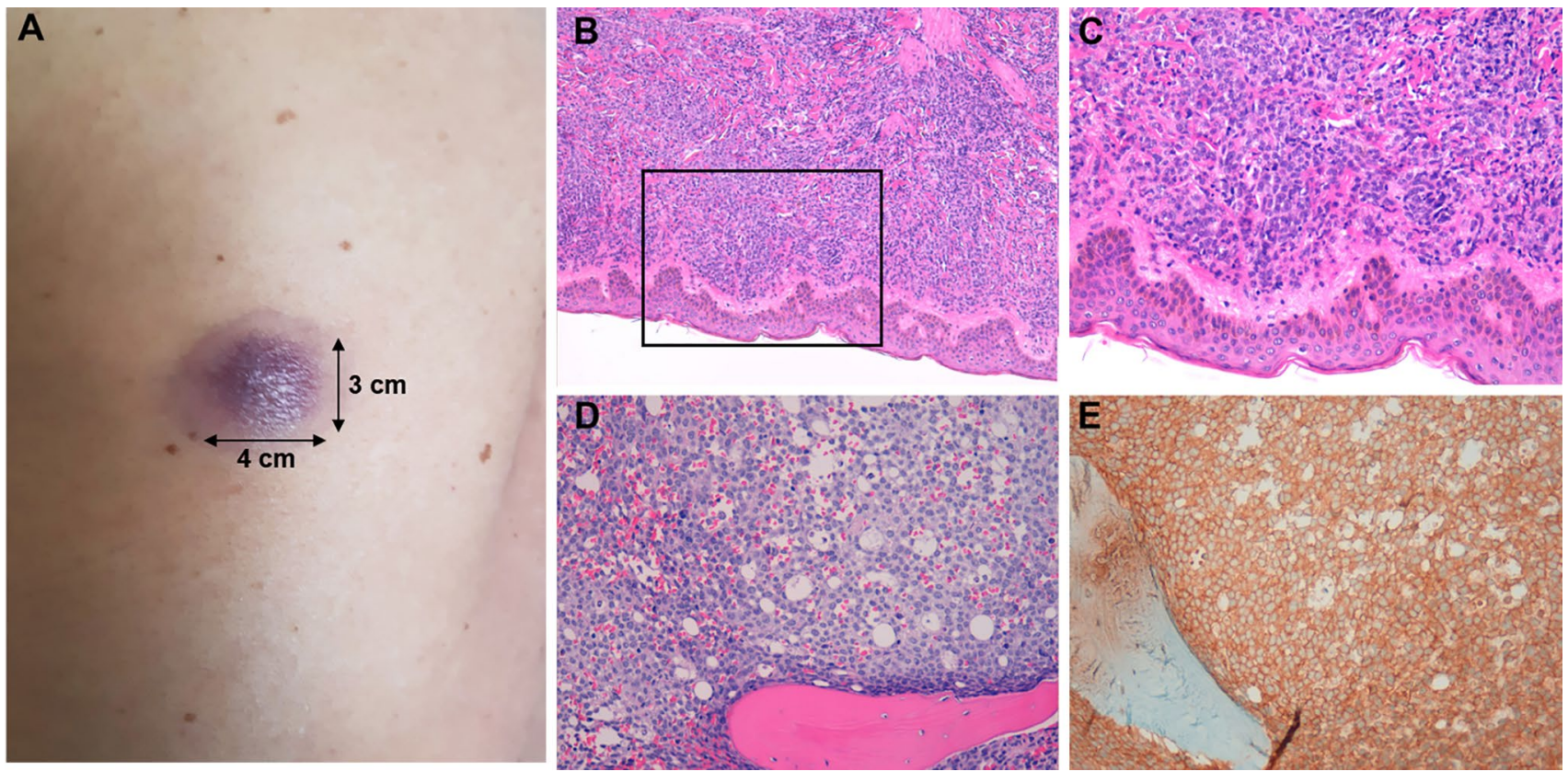

Fig. 1 Leukemic blasts infiltrate skin and bone marrow. A Macroscopic picture of a purple and thick plaque at the left scapula with B/C diffuse infiltration (90\%) of medium sized leukaemic blasts in respective histopathological examination of skin lesions. D H\&E morphology at intermediate magnification of bone marrow and $\mathbf{E}$ respective staining for the myeloid-specific blast marker CD33
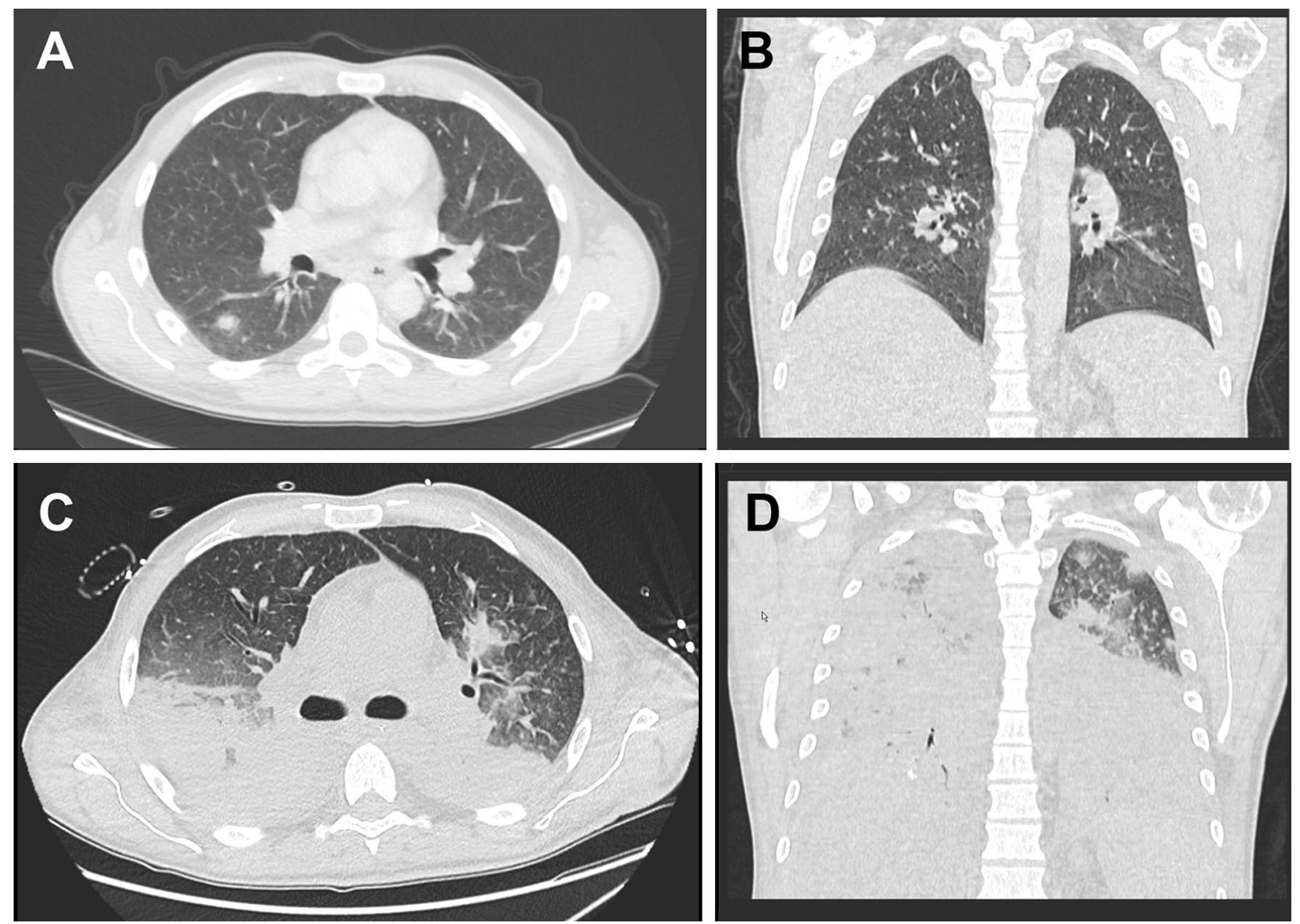

Fig. 2 CT images show pulmonary infiltrates initially presumed to be due to fungal infection or leukaemic manifestations at the time of admission $(\mathbf{A} / \mathbf{B})$ and progressive diffuse opacities due the development of severe ARDS 5 days later $(\mathbf{C} / \mathbf{D})$ 
AutOhor contributions Material preparation and data collection were performed by DCDS. The first draft of the manuscript was written by DCDS, LF and EK and all authors commented on previous versions of the manuscript. All authors read and approved the final manuscript.

Funding Open Access funding enabled and organized by Projekt DEAL. Not applicable.

Data availability Not applicable.

Code availability Not applicable.

\section{Declarations}

Conflict of interest DCDS, LF, MB and EK do not report any COI with the publication of this case.

Ethical approval Not applicable.

Consent to participate Verbal consent was obtained.

Consent for publication Verbal consent was obtained.

Open Access This article is licensed under a Creative Commons Attribution 4.0 International License, which permits use, sharing, adaptation, distribution and reproduction in any medium or format, as long as you give appropriate credit to the original author(s) and the source, provide a link to the Creative Commons licence, and indicate if changes were made. The images or other third party material in this article are included in the article's Creative Commons licence, unless indicated otherwise in a credit line to the material. If material is not included in the article's Creative Commons licence and your intended use is not permitted by statutory regulation or exceeds the permitted use, you will need to obtain permission directly from the copyright holder. To view a copy of this licence, visit http://creativecommons.org/licenses/by/4.0/.

\section{References}

1. Scussel R, et al. Macrolides or fluoroquinolones as enteral antibiotic therapy for non-ICU legionellosis. Infection. 2019;47(5):875-6.

2. Falcone M, et al. Predictors of intensive care unit admission in patients with Legionella pneumonia: role of the time to appropriate antibiotic therapy. Infection. 2021;49(2):321-5.

3. Lerolle N, et al. Pneumonia involving Legionella pneumophila and Listeria monocytogenes in an immunocompromised patient: an unusual coinfection. Respiration. 2002;69(4):359-61.

4. Goulet V, et al. Incidence of listeriosis and related mortality among groups at risk of acquiring listeriosis. Clin Infect Dis. 2012;54(5):652-60.

5. Wickramasekaran RN, Sorvillo F, Kuo T. Legionnaires' disease and associated comorbid conditions as causes of death in the US, 2000-2010. Public Health Rep. 2015;130(3):222-9. 